\title{
Computer-aided Kidney Segmentation on Abdominal CT Images Using Fuzzy Based Denoising for Gaussian Noise
}

\author{
Israt Jahan Tulin ${ }^{1, *}$, Md. Zaki Imam², Pronaya Prosun Das ${ }^{3}$ \\ ${ }^{1}$ Department of CSE, BGMEA University of Fashion and Technology, Bangladesh \\ ${ }^{2}$ Software Engineer Analyst, Accenture, Bangladesh \\ ${ }^{3}$ Department of CSE, Shanto-Mariam University of Creative Technology, Bangladesh
}

Copyright $\bigcirc 2017$ by authors, all rights reserved. Authors agree that this article remains permanently open access under the terms of the Creative Commons Attribution License 4.0 International License

\begin{abstract}
In this paper, we have proposed an image filtering technique (fuzzy logic based approach) to preserve important signal elements such as edges, smoothing the details of an abdominal CT image such as kidney to make it appear clear and sharpener. At first, abdominal CT images are retrieved from Gaussian noise using fuzzy logic based approach. After that, kidney region have been segmented from the abdomen area using region growing algorithm. In region growing process, the first stage detects the abdomen boundary using contour detection algorithm. The second stage identifies the kidney using seed point location. After successful segmentation, the kidney region is extracted and it is given to some region growing methods such as Region Growing Interest (ROI), pixel filling, erosion, labeling and dilation to find the accurate segmented area of these abdominal CT images. The results of a series of tests on 62 images from 16 patients indicate an interrelationship up to $73 \%$ between automatic and manual segmentation.
\end{abstract}

Keywords Gaussian Noise, Filter, CT Images, Kidney, Segmentation and Detection

General Terms Gaussian Noise, Image Filtering, Seed Point Searching, Kidney Segmentation, Detection and Region Growing Method

\section{Introduction}

Gaussian noise [1] is a type of statistical noise in which the amplitude of the noise follows that of a Gaussian distribution. When Images are Acquired and Transmitted over channels they are often corrupted by Impulse Noise [2,3] due to faulty communications and Noisy channels .i.e. Noise characterized by transient short-duration disturbances distributed essentially uniformly over the useful pass band of a transmission system. The Main aim of an Image De-noising Algorithm is to reduce the Noise Level, while keeping its fundamental structure like corners and edges [4]. Computed Tomography (CT) of an abdomen is an imaging method that uses x-rays and computer technology to produce images of the belly area. For diagnosis of abdominal organs pathologies, different imaging techniques such as Magnetic Resonance Imaging (MRI), Positron Emission Tomography, Computed Tomography (CT) and ultrasound scan are a standard instrument in medical field. Due to good spatial resolution and high Signal-to-Noise ratio Computed Tomography (CT) images are chosen to segment kidney region and detect healthy kidney images.

Image segmentation is the process of partitioning a digital image into multiple segments and the goal is to simplify or change the representation of an image into something that is more meaningful and easier to analyze.

Hence our main objective is to remove the Gaussian noise from the abdominal CT images and segment the kidney region from this abdominal area to make a size measurement or to characterize the difference between healthy versus diseased. It also may helpful to improve the speed and accuracy of further segmentation process.

\section{Gaussian Noise}

In many occasions, noise in digital images is found to be additive in nature with uniform power in the whole bandwidth with Gaussian probability distribution. Such a noise is referred to as additive white Gaussian noise (AWGN).The AWGN is mathematically represented by $\eta$,

$$
\begin{gathered}
\mathrm{N}_{\mathrm{AWGN}}(\mathrm{t})=\eta_{\mathrm{G}}(\mathrm{t}) \sigma \\
\mathrm{f}_{\mathrm{AWGN}}=\mathrm{f}(\mathrm{x}, \mathrm{y})+\eta_{\mathrm{G}(\mathrm{x}, \mathrm{y})}
\end{gathered}
$$

Where $\eta_{\mathrm{G}}(\mathrm{t})$ is a random variable that has a Gaussian probability distribution .It is an additive noise that is characters by its variance $\sigma^{2}$.In (5),the noisy image is represented as a sum of the original uncorrupted image and the Gaussian distributed random noise $\eta_{\mathrm{G}}$. When the variance of the random noise $\eta_{\mathrm{G}}$ is very low, $\eta_{\mathrm{G}(\mathrm{x}, \mathrm{y})}$ is zero or very 
close to zero at many pixel locations. Under such circumstances, the noisy image $\mathrm{f}_{\mathrm{AWGN}}$ is same or very close to the original image $\mathrm{f}(\mathrm{x}, \mathrm{y})$ at many pixel locations $(\mathrm{x}, \mathrm{y})$. Gaussian noise is generated during film exposure and development of the image. In this study each pixel in the output image is computed as a function of one or several pixels in the original image, usually located near the location of the output pixel $[5,6]$. The best solution for noise reduction or filtering is to process the image by its individual pixels based upon the appearance of its immediate neighbor pixels [7]. Image filtering improves the quality of the image by the way of enhancement of edges of the images. So many techniques such as mean filter, median filter, fuzzy filter and some other denoising techniques have been developed to suppress Gaussian noise [8-11]. Among all other filters, fuzzy image filter is very effective [12] and it can manage vague and uncertainty information efficiently.

\section{Literature Review}

Till now, many researchers have been focused for kidney segmentation. Pohle and Toennies developed a region-growing algorithm that automatically learns its homogeneity criterion from the characteristics of the region that is segmented [13-15]. This approach is less sensitive to the seed point location. Based on the pixel value distribution of the organ and the mesh operation [16,17], Kim et al. and Yoo et al. proposed similar approaches for kidney segmentation. To identify and extract organs from normal CT images [18], Kobashi and Shapiro explained a knowledge-based procedure. The detection result was rated $85 \%$ grade A from testing of 75 images from three patients. Through tissue texture analysis and distribution of directional maximums [19], Mavromatis et al. performed medical image segmentation. Wang et al. proposed a constrained optimization approach. In this process deformable contour can be computed as extra constraints within the contour energy minimization frame work [20]. Tsagaan and Shimizu described a deformable model approach for automatic kidney segmentation [21-23]. They used a deformable model represented by the grey level appearance of kidney and its statistical information of the shape. They tested about 33 abdominal CT images. The degree of correspondence between automatic segmentation and manual positioning was $86.9 \%$.

\section{Fuzzy Filtering Techniques}

Fuzzy set theory [24-26] has been successfully applied to pattern recognition fields. It is suitable for dealing with problems containing high levels of uncertainty, to which class pattern recognition or image processing problems usually belong. Obviously, the recovery of heavily noise-corrupted images is a task with high uncertainty levels. The general idea behind the filter is to average a pixel using other pixel values from its neighborhood, but simultaneously to take care of important image structures such as edges. The main concern of the present filter is to distinguish between local variations due to noise and due to image structure. In order to accomplish this, we derive a value for each pixel. This value expresses the degree in which the derivative in a certain direction is small. Such a value is derived by applying fuzzy rule [27-29] for each direction corresponding to the neighboring pixels of the processed pixel. The further construction of the filter is then based on the observation that a small fuzzy derivative $[25,26$,$] most likely is caused by$ noise, while a large fuzzy derivative most likely is caused by an edge in the image. Consequently, for each direction we will apply two fuzzy rules that take this observation into account (and thus distinguish between local variations due to noise and due to image structure), and that determine the contribution of the neighboring pixel values. The result of these rules (16 in total) is defuzzified and a "correction term" is obtained for the processed pixel value.

Two important features of the present filter are: first, the filter estimates a "derivative" in order to be less sensitive to local variations due to image structures such as edges; second, the membership functions are adapted accordingly to the noise level to perform smoothing operation.

Estimating derivatives and filtering are very complex as for filtering we need a good indication of the edges, while to find these edges we need filtering. To counter this problem, we start by looking for the edges. We try to provide a robust estimate by applying fuzzy rules.

Fuzzy image processing scheme is a collection of different fuzzy approaches to image processing [30]. It has three main stages. They are (i) Image fuzzification (ii) Membership modification (iii) Image defuzzification.

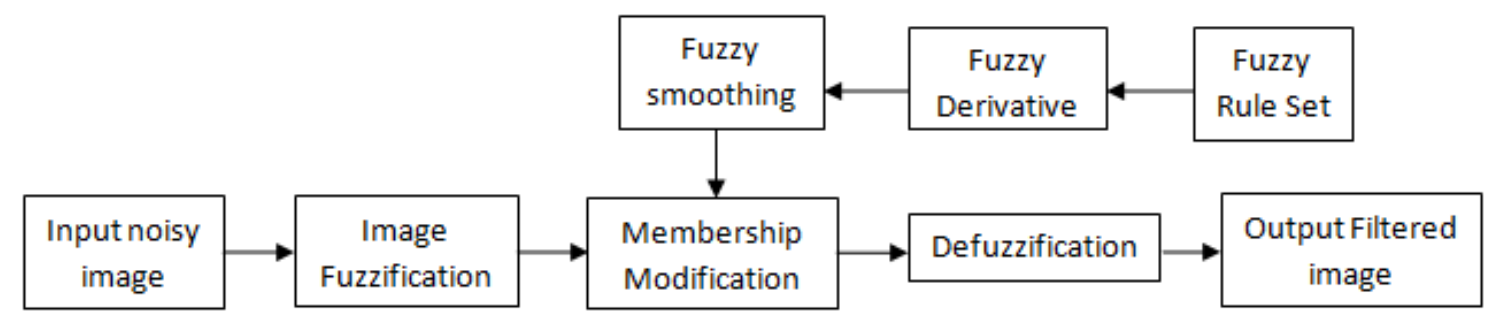

Figure 1. Basic structure of denoising image by using Fuzzy logic Algorithm 


\subsection{Grouped Mean Fuzzy Derivative}

By applying this algorithm, the gray values of the neighboring pixels ( $\mathrm{n} \times \mathrm{n}$ window) are stored in an array. It is then sorted in ascending or descending order. Then membership values are assigned to the neighborhood pixels. The neighborhood of a central pixel ( $x$ y) in $3 \times 3$ window of pixels is given by

\begin{tabular}{|c|c|c|}
\hline PNW & PN & PNE \\
\hline PW & PXY & PE \\
\hline PSW & PS & PSE \\
\hline
\end{tabular}

Figure 2. Pixels around a central pixel PXY

Now mean value of pixel values of PNW, PXY, PSE; PN, PXY, PS; PNE, PXY, PSW; PW, PXY, PE are calculated. After the calculation of the mean values for each group, grouped mean of all these groups is found. The pixel having the grouped mean, membership value ' 1 ' is assigned. The membership value ' 0 ' is assigned to the lowest and highest gray values. Now we consider only $2 \mathrm{x} \mathrm{k}+1$ pixel in the sorted pixels and they are the median gray values in the sorted list.

\subsection{Noise Estimation}

Consider a $3 \mathrm{X} 3$ neighborhood of a pixel (x, y). A simple derivative at the central pixel position $(\mathrm{x}, \mathrm{y})$ value in the direction $\mathrm{D}(\mathrm{D}=\mathrm{NW}, \mathrm{W}, \mathrm{SW}, \mathrm{S}, \mathrm{SE}, \mathrm{E}, \mathrm{NE}, \mathrm{N})$ is defined as the difference between the value of the central pixel $(x, y)$ and its neighbor pixel in that direction $\mathrm{D}$. This derivative value is denoted by $\tilde{N} d(x, y)$.

For example, $P N(x, y)=f(x, y-1)-f(x, y)$

The principle of the fuzzy derivative [31-33] is based on the following observation. Consider an edge passing through the neighborhood of a pixel $(x, y)$ in the direction SW, NE. The derivative value $\tilde{\operatorname{Nnw}}(\mathrm{x}, \mathrm{y})$ will be large, but also derivative values of neighboring pixels perpendicular to the edge's direction can expected to be large. For example, in NW - direction we can calculate the derivative values Nnw(x, $y), \tilde{N} n w(x-1, y+1)$ and $\tilde{N} n w(x+1, y-1)$. The idea is to cancel out the effect of one derivative value which turns out to be high due to noise. Therefore, if two out of three derivative values are small, it is safe to assume that no edge is present in the considered direction. This observation will be taken into account when we formulate the fuzzy rule to calculate the fuzzy derivative.

NW: (if $\tilde{N} n w(x, y)$ is small and $\tilde{N} n w(x-1, y+1)$ is small) or (if $\tilde{\operatorname{Nnw}}(\mathrm{x}, \mathrm{y})$ is small and $\tilde{\operatorname{Nnw}}(\mathrm{x}+1, \mathrm{y}-1)$ small) or (if $\tilde{\operatorname{Nnw}}(\mathrm{x} 1, \mathrm{y}+1)$ is small or $\tilde{\operatorname{Nnw}}(\mathrm{x}+1, \mathrm{y}-1)$ is small then $\tilde{\operatorname{Nnwf}}(\mathrm{x}$, y) is small

\subsection{Smoothing}

To compute the correction term $\nabla$ for the processed pixel value, we use a pair of fuzzy rules for each direction. The idea behind the rules is the following: if no edge is assumed to be present in a certain direction, the (crisp) derivative value in that direction can and will be used to compute the correction term. The first part (edge assumption) can be realized by using the fuzzy derivative value, for the second part (filtering) we will have to distinguish between positive and negative values. For example, let us consider the direction $N W$. Using the values $\nabla_{\mathrm{NW}}^{\mathrm{F}}(\mathrm{x}, \mathrm{y})$ and $\nabla_{\mathrm{NW}}(\mathrm{x}, \mathrm{y})$, we fire the following two rules, and compute their truthiness $\lambda_{\mathrm{NW}}^{+}$and $\lambda_{\mathrm{NW}}^{-}$:

$\lambda^{+}{ }_{\mathrm{NW}}$ : if $\nabla_{\mathrm{NW}}^{\mathrm{F}}(\mathrm{x}, \mathrm{y})$ is small and $\nabla_{\mathrm{NW}}(\mathrm{x}, \mathrm{y})$ is positive then $\mathrm{C}$ is positive

$\lambda_{\mathrm{NW}}$ : if $\nabla_{\mathrm{NW}}^{\mathrm{F}}(\mathrm{x}, \mathrm{y})$ is small and $\nabla_{\mathrm{NW}}(\mathrm{x}, \mathrm{y})$ is negative then $\mathrm{C}$ is negative.

For the properties positive and negative, we also use linear membership functions. Again, we implement the AND-operator and OR-operator by respectively the minimum and maximum.

The final step in the computation of the fuzzy filter is the defuzzification. We are interested in obtaining a correction term $\nabla$, which can be added to the pixel value of location ( $\mathrm{x}$, y).Therefore, the truthiness of the rules $\lambda^{+}{ }_{D}$ and $\lambda_{D}^{-}, D € \operatorname{dir}$ (so for all directions) are aggregated by computing and rescaling the mean truthiness as follows:

$$
\Delta=\frac{L}{8} \sum_{D € d i r}\left(\lambda_{D}^{+}-\lambda_{D}^{-}\right)
$$

Where dir contains the directions and $\mathrm{L}$ represents the number of gray levels. So, each direction contributes to the correction term $\Delta$.

The fuzzy rules for smoothing are as follows:

1. If a pixel is darker than neighboring pixels then make it brighter

2. If a pixel is brighter than neighboring pixels then make it darker.

3. Else leave it unchanged.

\begin{tabular}{|c|c|c|c|}
\hline & & $\begin{array}{c}(\mathrm{PN}) \mathrm{N} \\
\vee\end{array}$ & \\
\hline & $\begin{array}{c}\text { PNW } \\
\vee\end{array}$ & PN & $\begin{array}{c}\text { PNE } \\
v\end{array}$ \\
\hline $\begin{array}{c}(\mathrm{PW}) \mathrm{W} \\
v\end{array}$ & PW & P & PE \\
\hline & $\begin{array}{c}\text { PSW } \\
\vee\end{array}$ & PS & PSE \\
\hline
\end{tabular}

Figure 3. Estimation of the noise

\section{Image Quality Evaluation Matrix}

The filter performances are usually compared in term of PSNR and MSE. Larger values of PSNR and small values of MSE indicate less noise power in an image.

\section{Mean Square Error (MSE)}

MSE is given by

$$
M S E=\frac{1}{M N} \sum_{X=1}^{M} \sum_{y=1}^{N}\left[\mathrm{f}(\mathrm{x}, \mathrm{y})-\mathrm{f}^{\prime}(\mathrm{x}, \mathrm{y})\right]^{2}
$$


Where $f(x, y)-M x N$ initial image

$f(x, y)$ - Noised image

\section{Peak Signal Noise Ratio (PSNR)}

PSNR is defined as

PSNR $=10 \log _{10}\left(255^{2} / \mathrm{MSE}\right) \mathrm{dB}$.

If PSNR value is high or MSE value is low THEN image quality is better. Filtering techniques are as follows:

Table 1. Comparison of the MSE'S and PSNR of restored images between different images

\begin{tabular}{|c|c|c|}
\hline Filter & MSE & PSNR \\
\hline Mean filter & 372 & $18.05 \mathrm{db}$ \\
\hline Median filter & 225 & $20.07 \mathrm{db}$ \\
\hline Proposed filtering technique & 99 & $26.09 \mathrm{db}$ \\
\hline
\end{tabular}

\subsection{Experimented Result}

In this study, different noise reduction techniques are tested on various standard CT images and found that fuzzy filter is the effective algorithms which gives the better results in terms of Visual Quality, high speed etc. To compare the performance of various noise removal approaches, these different methods have been repeated several times and the results evaluated using MSE and PSNR criteria.

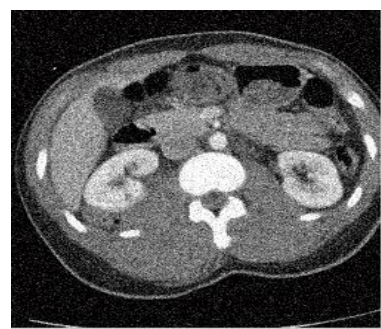

Noisy Image

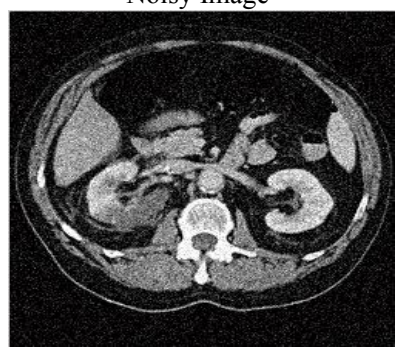

Noisy Image

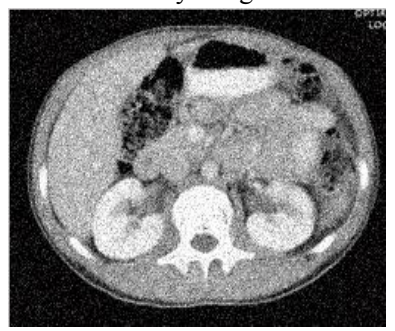

Noisy Image

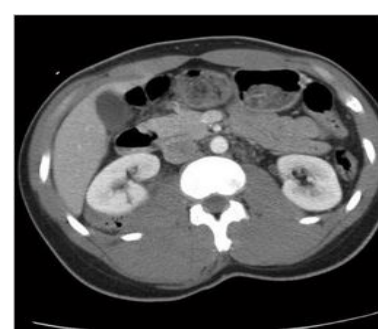

Filtered Image

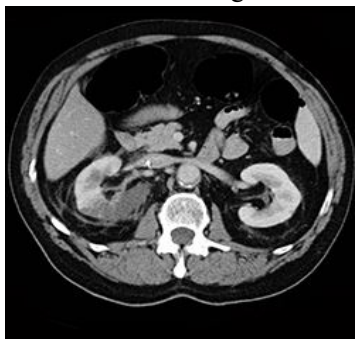

Filtered Image

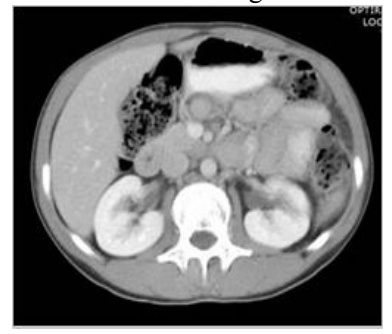

Filtered Image
Figure 4. Gaussian noise removals using fuzzy based filter

\section{Kidney Segmentation}

An abdominal CT image is very complicated. It contains kidney, liver, spleen, spine, fat, and pathologies. To segment the kidney region from the abdominal CT images, at first we have applied an abdomen contour detection algorithm to delineate the abdominal cavity. After successfully determining the abdominal boundary, we have segmented kidney region by applying region growing segmentation algorithm.

\subsection{Region Growing Process}

Region growing algorithm is a pixel-based image segmentation method. It involves the selection of initial seed points. This algorithm works with a set of seed points. Each seed points correspond to an individual region. The seed points compare to their neighboring pixels based on a similarity criterion. These neighboring pixels are then computed either by 4-connectivity or 8-connectivity. The neighboring pixels in 4-connectivity are connected horizontally and vertically, whether the neighboring pixels in 8-connectivity are connected horizontally, vertically, and diagonally. The simplest similarity criteria are used generally. It can be obtained by calculating the difference between the intensity value of the image pixel and the corresponding region mean. If the difference is less than a specified threshold, then the pixel belongs to that region and is subsequently labeled. Otherwise the pixel is not labeled and skipped.

\subsection{Region Growing Algorithm}

Step 1: Every seed point corresponds to a single region and by applying the seed selection algorithm, these seed points are computed.

Step 2: For a given seed point,

- Seed point is assigned as the region label.

- Initialize the region mean and it is equal to the pixel intensity at the seed point.

- Neighbours of the seed point are computed and stored in the neighbour matrix. This matrix stores the neighboring pixels addresses, to be checked.

Step 3: For every pixel stored in the neighbour matrix,

- The pixel which is not labeled and fulfill the similar criteria,

I The pixel in the corresponding region is labeled.

II The new region mean of the corresponding region is computed.

III The neighboring of pixels are computed and stored in the neighbour matrix.

- Otherwise, skip the pixel and choose the next neighboring pixel.

- $\quad$ Repeat step 3, until all the listed neighboring pixels are not checked. 
- Leave the region pixels unlabelled, if the region size is very small.

Step 4: The next seed point is chosen and repeat step 2 for next region.

Step 5: If all the pixels are not labeled, the seed points are computed again using only the unlabelled pixels.

Step 6: Repeat the process until all pixels of the image are labeled into their corresponding regions. In this way, the image is divided into various segmented regions.

\subsection{Region Modification}

After applying the region growing method to the abdomen area we have obtained different gray scale images of kidney region. But it may remain some trivial and irregular objects or holes that may scatter inside the region. So at first we have applied ROI (Region Growing Interest) process to find a portion of an image that we want to filter. After that we have applied a series of image processing skills such as pixel filling, erosion, labeling and dilation to improve the accuracy of the segmented area under various conditions arising from clinical practices such as the timing and rate of contrast media injection, image intensity variations, and blood flow rate etc.

\subsection{Experiment Result}

After removing the Gaussian noise from the abdominal area of CT images, these renal areas have been segmented through region growing methods.

The segmented regions have been found in binary form. Then we have converted these to gray scale images.

\subsubsection{Kidney Segmentation}
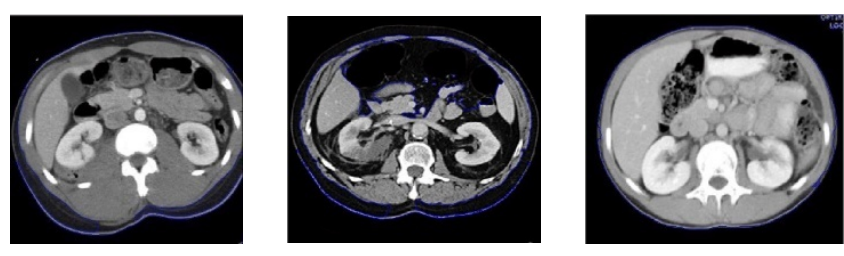

Figure 5. Detection of abdominal boundary
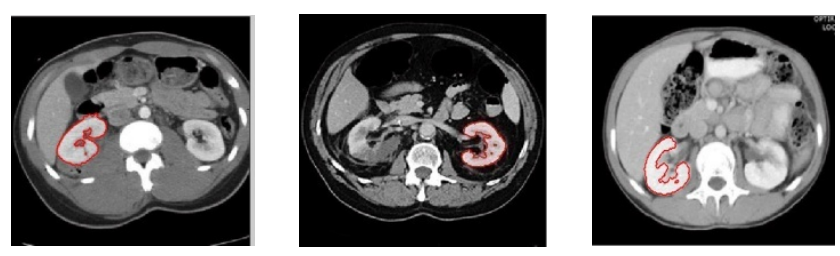

Figure 6. Kidney extraction
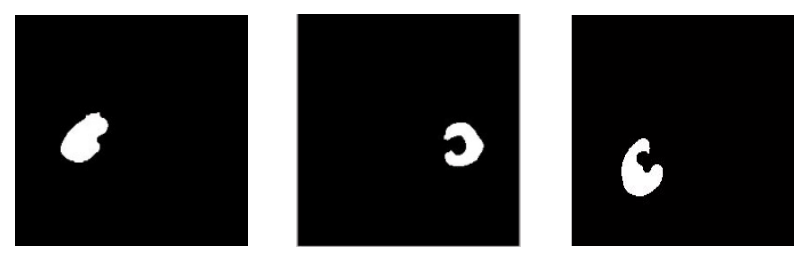

Figure 7. Kidney Segmentation

6.4.2. Region Modification
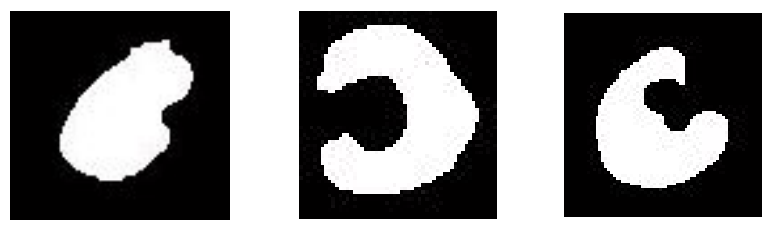

Figure 8. Binary Images
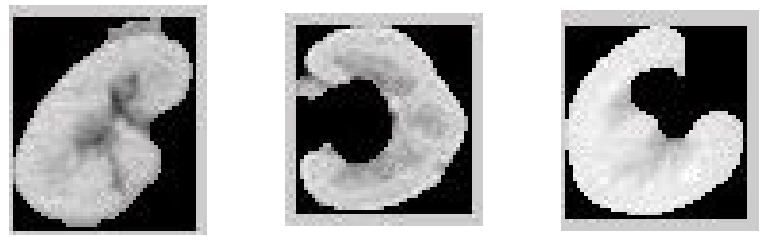

Figure 9. Gray Scale Images

\section{Conclusions}

In this Paper using correction Term Method, we have introduced a new algorithm for de-noising images and also used region growing method to segment abdominal CT images. The implementation of our fuzzy Filter [34-37] in the image restoration process was discussed. All the techniques and operations provide an efficient working.

It presents a new technique for filtering narrow-tailed and medium narrow-tailed noise by a fuzzy filter [34, 35, 37]. The existing system is available for reducing fat-tailed noise like impulse noise [38, 39]. Median filtk6er [40-41] and Low-pass filters are in job at present. Median Filters [41, 42] mainly concentrate only on Impulsive Noise. Gaussian Noise is not specifically concentrated. It does not distinguish local variation due to noise and due to image structure proposed filter can clean an image completely of noise without making it blurry.

In the future work, this proposed fuzzy filter will be focused on improving the system performance for both gray scale and color images. 


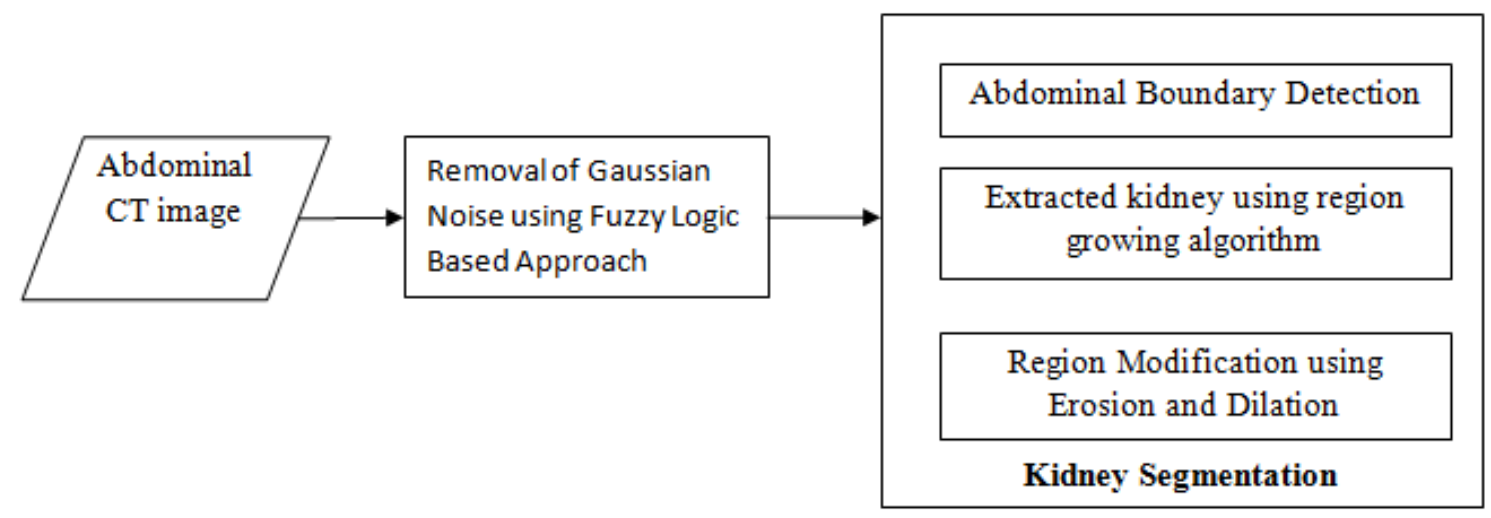

Figure 10. Overall methodology of our proposed approach

\section{Acknowledgements}

At first author offers gratitude to Almighty Allah who gave the ability to complete the whole thesis work without any breaking.

Author was partially supported by the Department of Computer Science and Engineering, Jahangirnagar University, Savar, Dhaka, Bangladesh.

Author would also like to express gratitude to all the respected colleagues \& all staffs of BUFT (BGMEA University of Fashion and Technology) who helped in different ways for this research project.

Lastly author wants to express hearty complement to the parents \& family members for their constantly encouragement to come at this stage.

\section{REFERENCES}

[1] "A fuzzy filter for images corrupted by impulse noise," IEEE Signal Processing Lett., vol. 3, pp. 168-170, June 1996.

[2] C.-S. Lee and Y.-H. Kuo, Fuzzy Techniques in Image Processing. New York: Springer-Verlag, 2000, vol. 52, Studies in Fuzziness and Soft Computing, ch. Adaptive fuzzy filter and its application to image enhancement, pp. 172-193.

[3] F. Russo, "Fire operators for image processing," Fuzzy Sets Syst., vol. 103, no. 2, pp. 265-275, 1999.

[4] C.-S. Lee, Y.-H. Kuo, and P.-T. Yu, "Weighted fuzzy mean filters for image processing," Fuzzy Sets Syst., no. 89, pp. 157-180, 1997.

[5] H. C. Andrews, A. G. Tescher and R. P. Kruger, "Image processing by digital computer", IEEE spectrum, Vol. 9, pp 20-32, July 1972.

[6] R. Gonzalez and P. Wintz, Digital image processing, Addison - Wesley, 1987.

[7] Lee, C. S., V. H. Kuo and P. T. Yu 1997. Weighted fuzzy mean filters for image processing, Fuzzy Sets. Sys., 89, $157-$ 180.

[8] T. Zong, H. Lin and T. Kao, "Adaptive local contrast enhancement method for medical images displayed on a video monitor, "Med Eng. Phys., Vol.22, pp-79-87, 2000.

[9] A. Polesel, G. Ranponi and V. J. Mathews, "Image enhancement via adaptive unsharp masking". IEEE Trans. Image Processing, vol.9, pp 505-510, Mar. 2000.

[10] S. S. Agaian, K. Panetta and A. M. Grigoryan, "Transform based image enhancement algorithms with performance measure", IEEE Trans. Image processing Vol.10, pp 367-382 Mar. 2001.

[11] M. Nachtegael, D. Wicken, D Ville, E. Korre, "Studies in Fuzziness and soft computing: Fuzzy filters for image Processing”. Springer 2003, pp 28-29.

[12] E. Kare and M. Nachtegael, Eds., "Fuzzy techniques in image processing", New York: Springer - Verlag 2000, Vol. 52. Studies in Fuzziness and soft computing.

[13] E. Supriyanto, Hafizah W. M, Wui Y. J, Arooj A. Automatic Non Invasive Kidney Volume Measurement Based On Ultrasound Image Proceedings of 15th International WSEAS GSCC Multi conferences held in Corfu Island, Greece, July 14-16, 2011 ISBN: 978-1-61804-019-0 Page 387-392

[14] R. Pohle and K. D. Toennies, "A new approach for model-based adaptive region growing in medical image analysis," in the 9th Int. Conf. Computer Analysis of Images and Patterns, vol. 2124, Warsaw, Poland, 2001, pp. 238-246.

[15] R. Pohle and K. D. Toennies, "Segmentation of medical images using adaptive region growing," in Proc. Int. Soc. Opt. Eng. (SPIE), vol. 4322, 2001, pp. 1337-1346.

[16] R. Pohle and K. D. Toennies, "Self-learning model-based segmentation of medical images," Image Process. Commun. vol. 7, pp. 97-113, 2001.

[17] S. H. Kim, S. W. Yoo, S. J. Kim, J. C. Kim, and J. W. Park, "Segmentation of kidney without us in gcontrast medium on abdominal CT image," in 5th Int. Conf. Signal Processing, vol.2, Beijing, China, 2000, pp. 1147-1152.

[18] S. W. Yoo, J. S. Cho, S. M. Noh, K. S. Shin, and J. W. Park, "Organ segmentation by comparing of gray value portion on abdominal CT image," in 5th Int. Conf. on Signal Processing, vol. 2, Beijing, China, 2000, pp. 1201-1208.

[19] M. Kobashi and L. G. Shapiro, "Knowledge-based organ identification from CT images," Pattern Recogn., vol. 28, pp. 475-491, 1995. 
[20] S. Mavromatis, J.M. Bo, and J. Sequeira, "Medical image segmentation using texture directional features," in IEEE 23rd Annu. Int. Conf. Eng. Med. Biol. Soc., vol. 3, Istanbul, Turkey, 2001

[21] X. Wang, L. He, and W. G. Wee, "Deformable contour method: A constrained optimization approach," Int. J. Comput. Vision, vol. 59, no. 1, pp. 87-108, 2004.

[22] B. Tsagaan, A. Shimizu, H. Kobatake, K. Miyakawa, and Y. Hanzawa, "Segmentation of kidney by using a deformable model," in Int. Conf. Image Processing, vol. 3, Thessaloniki, Greece, 2001, pp. 1059-1062.

[23] B. Tsagaan, A. Shimizu, H. Kobatake, and K. Miyakawa, “An automated segmentation method of kidney using statistical information," in Proc. Medical Image Computing and Computer Assisted Intervention, vol. 1, 2002, pp. 556-563.

[24] . Nedeljkovic, «Image Classification Based On Fuzzy Logic». The International Archives of the Photogrammetry, Remote Sensing and Spatial Information Sciences, Vol. 34, 2004.

[25] H.S. Kam, M. Hanmandlu, and W.H. Tan, “An adaptive fuzzy filter system for smoothing noisy images", Proc. of Intl. Conf. on Convergent Technologies for Asia-Pacific Region, Vol.4, pp. $1614-1617,2003$.

[26] Ville D., Nachtegael M., Weken D., Kerre E., Philips W., and Lemahieu I., "Noise Reduction by Fuzzy Image Filtering", IEEE Transactions on Fuzzy system, Vol. II, No.4, August, 2003.

[27] D. Van De Ville, M. Nachtegael, D. Van der Weken, E. Kerre, W.Philips, and I. Lemahieu, Noise Reduction by Fuzzy Image Filtering, IEEE transactions on fuzzy systems, vol. 11, No. 4, August 2003.

[28] K. Arakawa, Median filter based on fuzzy rules and its application to image restoration, Fuzzy Sets Syst., pp. 3-13, 1996.

[29] F. Russo and G. Ramponi, A fuzzy operator for the enhancement of blurred and noisy images, IEEE Trans. Image Processing, vol. 4, pp.1169-1174, August 1995.

[30] Mario. I., Chacon. M, "Fuzzy logic for image processing", Advanced fuzzy logic techniques in industrial applications", 2006.

[31] C.-S. Lee and Y.-H. Kuo, Fuzzy Techniques in Image Processing. New York: Springer-Verlag, 2000, vol. 52, Studies in Fuzziness and Soft Computing, ch. Adaptive fuzzy filter and its application to image enhancement, pp. 172-193.
[32] C.-S. Lee, Y.-H. Kuo, and P.-T. Yu, "Weighted fuzzy mean filters for image processing," Fuzzy Sets Syst., no. 89, pp. 157-180, 1997.

[33] Wang, Z.[Zhou], Zhang, D., Restoration of Impulse Noise-Corrupted Images Using Long-Range Correlation, IEEE Signal Processing Letters , (5), No. 1, January1998, pp. 4-7. 9802 [14] Russo, F., Ramponi, G., A Fuzzy Filter for Images Corrupted By Impulse Noise, IEEE Signal Processing Letters, (3), No. 6, June 1996, pp. 168-170. 9607

[34] I. Nedeljkovic, « Image Classification Based On Fuzzy Logic». The International Archives of the Photogrammetry, Remote Sensing and Spatial Information Sciences, Vol. 34, 2004.

[35] H. S. Kam, M. Hanmandlu, and W. H. Tan, "An adaptive fuzzy filter system for smoothing noisy images", Proc. of Intl. Conf. on Convergent Technologies for Asia-Pacific Region, Vol.4,pp. 1614 - 1617, 2003.

[36] Ville D., Nachtegael M., Weken D., Kerre E., Philips W., and Lemahieu I., "Noise Reduction by Fuzzy Image Filtering", IEEE Transactions on Fuzzy system, Vol. II, No.4, August, 2003.

[37] D. Van De Ville, M. Nachtegael, D. Van der Weken, E. Kerre, W. Philips, and I. Lemahieu, Noise Reduction by Fuzzy Image Filtering, IEEE transactions on fuzzy systems, vol. 11, No. 4, August 2003.

[38] Stefan Schulte, Mike Nachtegael, Valerie de Witte, Dietrich van der Weken and Etienne E.Kerre, "A fuzzy Impulse noise detection and reduction method", IEEE Trans. on Image Process, Vol. 15,no 5,May 2006.

[39] M. Emin Yuksel, “A Hybrid Neuro-Fuzzy filter for Edge Preserving Restoration of Images Corrupted by Impulse Noise", IEEE Trans. on Image process, Vol.15, no 6, April 2006.

[40] P-E. Ng and K-K. Ma, "A Switching Median Filter with Boundary Discriminative Noise detection for Extremely Corrupted Images", IEEE Trans. on Image Processing, Vol. 15, No. 6, June 2006

[41] J. Ishihara, M. Meguro and N. Hamada, "Adaptive weighted median filter utilizing impulsive noise detection," in Applications of Digital Image Processing '99, Proc. SPIE 3808, 406-414 (1999)

[42] Chen, C. T. [Chun-Te], Chen, L. G. [Liang-Gee], A Self-Adjusting Weighted Median Filter for Removing Impulse Noise in Images, ICIP96(16A9). BibRef 9600 Proceedings International Conference on Image Processing, or ICIP. Sponsored by IEEE Signal Processing. 